\title{
Challenges facing effective implementation of co- trimoxazole prophylaxis in children born to HIV- infected mothers in the public health facilities
}

This article was published in the following Dove Press journal:

Drug, Healthcare and Patient Safety

29 October 2015

Number of times this article has been viewed

\author{
Appolinary AR Kamuhabwa' \\ Vicky Manyanga ${ }^{2}$ \\ 'Unit of Pharmacology and \\ Therapeutics, ${ }^{2}$ Department of \\ Medicinal Chemistry, School of \\ Pharmacy, Muhimbili University of \\ Health and Allied Sciences, Dar es \\ Salaam, Tanzania
}

Background: If children born to HIV-infected mothers are not identified early, approximately $30 \%$ of them will die within the first year of life due to opportunistic infections. In order to prevent morbidity and mortality due to opportunistic infections in children, the World Health Organization recommends the use of prophylaxis using co-trimoxazole. However, the challenges affecting effective implementation of this policy in Tanzania have not been documented.

Aim: In this study, we assessed the challenges facing the provision of co-trimoxazole prophylaxis among children born to HIV-infected mothers in the public hospitals of Dar es Salaam, Tanzania.

Methodology: Four hundred and ninety-eight infants' PMTCT (Prevention of Mother-to-Child Transmission of HIV) register books for the past 2 years were reviewed to obtain information regarding the provision of co-trimoxazole prophylaxis. One hundred and twenty-six health care workers were interviewed to identify success stories and challenges in the provision of co-trimoxazole prophylaxis in children. In addition, 321 parents and guardians of children born to HIV-infected mothers were interviewed in the health facilities.

Results: Approximately $80 \%$ of children were initiated with co-trimoxazole prophylaxis within 2 months after birth. Two hundred and ninety-one (58.4\%) children started using co-trimoxazole within 4 weeks after birth. Majority ( $\mathrm{n}=458,91.8 \%$ ) of the children were prescribed $120 \mathrm{mg}$ of co-trimoxazole per day, whereas $39(7.8 \%)$ received $240 \mathrm{mg}$ per day. Only a small proportion $(\mathrm{n}=1,0.2 \%$ ) of children received $480 \mathrm{mg} /$ day. Dose determination was based on the child's age rather than body weight. Parents and guardians reported that $42(13.1 \%)$ children had missed one or more doses of co-trimoxazole during the course of prophylaxis. The majority of health care workers $(89.7 \%)$ reported that co-trimoxazole is very effective for the prevention of opportunistic infections among children, but frequent shortage of co-trimoxazole in the health facilities was the main challenge.

Conclusion: Most children who were initiated with co-trimoxazole prophylaxis did not experience significant opportunistic infections, and the drug was well tolerated. The major barrier for co-trimoxazole prophylaxis was due to frequent out-of-stocks of pediatric co-trimoxazole formulations in the health facilities. Dose determination was based on the age rather than the weight of children, thus creating potential for under- or over-dosing of children.

Keywords: health facilities, opportunistic infections, CD4, parents, guardians

\section{Introduction}

In 2012, approximately 1.4 million people were living with HIV in Tanzania. ${ }^{1}$ The HIV population among the children under 5 years is estimated to be 66,233 (31,659 males and 34,574 females). The major cause of HIV in children is the transmission of the virus from the mother to the child in utero, during labor, delivery, or breast-feeding. 
Due to immature immune system, HIV-infected children are more prone to opportunistic infections than adults. Therefore, to prevent opportunistic infections during the early years of life, especially when the serostatus of the child is not yet confirmed, the use of antibiotic prophylaxis is recommended. ${ }^{2}$

Pneumocystis jirovecii pneumonia (PCP) is the most common opportunistic infection in children infected with HIV, causing high morbidity and mortality in children. ${ }^{3}$ More than $50 \%$ of all cases of PCP in children who acquire HIV from their mothers occur in infants of younger age ( $\leq 6$ months). ${ }^{4}$ It is therefore necessary that these infants are identified early so that prophylactic therapy can be started.

Co-trimoxazole is the combination of sulfamethoxazole and trimethoprim in the ratio of $5: 1 .^{5}$ Trimethoprim and sulfamethoxazole have enhanced effect when used concomitantly. This is because they inhibit sequential stages in the folate synthesis pathway of the microorganisms. Sulfamethoxazole works as a false-substrate to inhibit dihydropteroate synthetase. On the other hand, trimethoprim inhibits the action of bacterial dihydrofolate reductase, resulting in the inhibition of tetrahydrofolic acid synthesis. Since bacteria are unable to take up folic acid from the infected host, they depend on their own synthesis of folic acid. Due to inhibition of these two enzymes by co-trimoxazole, the bacteria are devoid of the important bases for DNA replication and transcription. ${ }^{6}$

In developed countries, co-trimoxazole prophylaxis is a standard of care for people living with HIV and AIDS, and is used in most of the African countries for the management of opportunistic infections. Evidence from trials on co-trimoxazole prophylaxis conducted in African countries show reductions in mortality between $25 \%$ and $46 \%$, and a beneficial effect of co-trimoxazole in reducing morbidity in people living with HIV and AIDS. ${ }^{7,8}$ The primary benefit of co-trimoxazole prophylaxis has been in the reduction of the incidence of PCP. ${ }^{3}$ It also decreases the rates of bacterial pneumonia, toxoplasmosis, and other infections, including reducing incidences of malaria and non-typhoid salmonellosis and diarrhea. ${ }^{7,9}$

Establishing whether an infant born to HIV-infected mother is also infected with the virus cannot be confirmed using antigen-antibody reaction tests. This is due to the presence of maternal antibodies in infants in the early months after birth. Unless advanced diagnostic techniques such as polymerase chain reaction (PCR) are available, presumptive preventive therapy using co-trimoxazole is an economical and feasible strategy, especially in resource-poor countries. ${ }^{10}$ Due to logistical and diagnostic difficulties in resource-poor settings, co-trimoxazole prophylaxis before confirmation of the serostatus of the child is necessary for at least 12 months to ensure prevention of opportunistic infections in those already infected with HIV. ${ }^{11}$ However, this strategy also has its limitations in resource-limited settings, with potential for increased bacterial resistance due to improper use of the drug. ${ }^{12}$

Due to the proven effectiveness and safety of cotrimoxazole for the management of opportunistic infections in children, World Health Organization recommends that all children born to HIV-infected mothers should be provided with presumptive prophylaxis during 4-6 weeks after birth. ${ }^{10}$ In the absence of PCR, prophylaxis should be continued for at least 1 year, when it can be confirmed that the child is not HIV-infected and no longer exposed to HIV from the mother. These guidelines have also been adopted by the Tanzanian National AIDS Control Program for the management of children born to HIV-infected mothers. ${ }^{13}$

Although countries have adapted the World Health Organization policy for co-trimoxazole prophylaxis, it is estimated that in the year 2008 , only $8 \%$ of children born to HIV-infected mothers were initiated co-trimoxazole prophylaxis by 2 months of age. ${ }^{14}$ Identification of children who are eligible for co-trimoxazole is a main challenge in most of the settings. The main reason for this may be due to inadequate training of health care workers, unavailability of diagnostic facilities, and lack of awareness and sensitization of parents and guardians of children. ${ }^{15}$

Other limitations for effective implementation of this policy may include the unavailability of essential medicines including co-trimoxazole in the health facilities. ${ }^{16}$ Therefore, understanding of the challenges encountered in the implementation of co-trimoxazole prophylaxis is necessary for the successful use of the drug. In this study, we assessed the level of the implementation of this policy in the public health facilities in Dar es Salaam, Tanzania, and identified the most common challenges from a health facility, parents/guardians, and health care workers perspectives.

\section{Patients and methods}

\section{Study area}

The study was conducted in the PMTCT (Prevention of Mother-to-Child Transmission of HIV) units at the care and treatment centers (CTCs) in the public hospitals in Dar es Salaam between August 2013 and March 2014. The study was conducted in four public hospitals (Muhimbili National Hospital, Mwananyamala, Temeke, and Amana Regional Hospital). 


\section{Study design}

It was both a retrospective and descriptive study that involved review of the records of infants from PMTCT register books of at least past 2 years, and interviewing health care workers and parents or guardians of the infants at the CTCs.

\section{Sample size and sampling technique Infant's record files}

The recommended age for initiation of co-trimoxazole for prophylaxis of opportunistic infections in children born to HIV-infected mothers is $4-6$ weeks of age. ${ }^{10,13}$ The review of PMTCT register books in a pilot study conducted at the Pastoral Activities and Services for People with AIDS in Dar es Salaam Archdiocese (PASADA) in 2012 showed that approximately $68 \%$ of infants were initiated cotrimoxazole prophylaxis at the age of 6 weeks. In addition, it was observed that children of up to 30 months had not yet determined their HIV status and were still being provided with co-trimoxazole prophylaxis. In this study, children of up to 36 months whose records had been taken for at least 24 months were included. Therefore, for calculation of sample size, $68 \%$ was used as a proportion of infants estimated to have been initiated co-trimoxazole prophylaxis at the age of 6 weeks. The calculated sample size was 335 infants' register books. Based on the pilot study conducted at PASADA, it was observed that up to $20 \%$ of the infant files had insufficient records for the intended study objectives. Therefore, the calculated sample size was adjusted upward to 498 .

The number of infants from each hospital was obtained by using a systematic sampling technique. Names of infants in the PMTCT register books were assigned numbers. They were then systematically selected by consecutively picking the infants assigned with even numbers until the sample size of infant's register books was reached in each hospital. Proportion of infants selected from each hospital had taken into account the number of infants registered at the PMTCT unit of the health facility. Based on this, 200 infants' files were selected from Muhimbili National Hospital, 109 from Mwananyamala hospital, 90 from Temeke hospital, and 99 files were from Amana hospital. A designed form was used for the collection of the required information from infants' register books in these facilities.

The standards that were used to guide data collection are in accordance with the Tanzanian National AIDS Control Program guidelines. ${ }^{13}$ In these guidelines, it is stated that all the children born to HIV-infected mothers should be given co-trimoxazole prophylaxis to prevent them from opportunistic infections. The recommended daily dosing of co-trimoxazole is shown in Table 1.

Follow-up of HIV-exposed children should be done every month for up to 1 year; then for every 3 months up to 5 years. Co-trimoxazole prophylaxis should be started from 4 to 6 weeks according to the dosage indicated in Table 1 . Documentation on tolerance and adherence to co-trimoxazole is done at every visit at the CTC. To confirm the serostatus of the child, antibody test for HIV infection should be done at 18 months. If the child is breast-feeding, antibody test for HIV infection should be done at 6 weeks after stopping breast-feeding. If PCR is available in the health facility, it is recommended to perform HIV test after 6 weeks.

\section{Interview of parents and guardians of children at the health facilities}

A total of 321 parents and guardians attending CTCs with their children were interviewed about their perceptions, adherence to co-trimoxazole prophylaxis and challenges encountered during the provision of co-trimoxazole prophylaxis to their children. The number of parents and guardians selected for interview in the selected health facilities was obtained by using a statistical formula to get the required minimum sample size. Selection of parents and guardians for interviews with the health facilities was then made through convenient sampling. That is, parents or guardians meeting the inclusion criteria attending the clinics during the study period were recruited in the study for interviews. The reasons for using convenient sampling to enroll parents and guardians in the study was mainly due to inadequate number of parents and guardians, who were attending antenatal clinics, met inclusion criteria and were willing to participate in the study. Eighty-seven parents and guardians were interviewed at Muhimbili National Hospital, while 78 parents and guardians were interviewed from each of the three regional hospitals, ie, Mwananyamala, Amana, and Temeke.

\section{Health care workers}

Health care workers who were interviewed in this study, were directly involved in the management of children with

Table I Recommended daily dosage of co-trimoxazole for prophylaxis of opportunistic infections in children born to HIVinfected mothers

\begin{tabular}{|c|c|}
\hline Weight of the child (kg) & $\begin{array}{l}\text { Dosage and formulation of } \\
\text { co-trimoxazole }\end{array}$ \\
\hline Below 10 & $5 \mathrm{mg} / \mathrm{kg}$ syrup \\
\hline Between 10 and 15 & $1 / 2$ tablet single strength* \\
\hline Above 15 & 2 tablets single strength* \\
\hline
\end{tabular}


HIV/AIDS or those born to HIV-infected mothers. ${ }^{17}$ A recent survey that we conducted in the selected public hospitals in Dar es Salaam showed that there were few health care workers who are directly involved in the management of children living with HIV/AIDS and those born to HIV-infected mothers whose serostatus have not yet been confirmed. The number of health care workers selected for interview in the health facilities was obtained by using a statistical formula to get the required minimum sample size. Due to the limited number of health care workers in the public health facilities, selection for interviews was done using convenient sampling for those who were present at the health facilities during the time of the study. In total, 126 health care workers including prescribers (medical doctors, assistant medical officers, and clinical officers), drug dispensers (pharmacists and pharmaceutical technicians), nurses, and counselors were interviewed.

\section{Data collection method}

Data were collected by using a design form to gather information of infants from the PMTCT register books. The data were collected through the review of the PMTCT register books to document the information on co-trimoxazole prophylaxis including the doses given, duration, timing on the establishment of HIV status of the child and patient follow-up for drug tolerance and adherence.

Questionnaires were used to interview parents, guardians, and health care workers regarding the use of co-trimoxazole for prophylaxis of opportunistic infections in children. For interviews, data were collected using structured questionnaires containing open and closed-ended questions. Research assistants conducted interviews with parents and guardians attending CTCs at the selected health facilities during their routine appointments or when visiting the pharmacies for co-trimoxazole prescription refill. Similarly, research assistants conducted interviews with health care workers at their work stations. Prior to data collection, research assistants had training for 2 days on research procedures and familiarization with data collection tools.

\section{Data analysis}

Data analysis was done using Statistical Package for Social Sciences (SPSS version 20; IBM Corporation, Armonk, NY, USA) computer software. Measure of central tendency and dispersion were analyzed using descriptive statistics. The association between the provision of co-trimoxazole prophylaxis in line with standard treatment guidelines and other factors (such as socio-demographic characteristics of health care workers, parents/guardians; availability of co-trimoxazole pediatric formulations; the proportion of infants born to HIVinfected mothers initiated co-trimoxazole prophylaxis as per the national treatment guidelines; doses of co-trimoxazole prescribed to children for prophylaxis of opportunistic infections; and duration of co-trimoxazole prophylaxis in infants born to HIV-infected mothers) were assessed using chi-square and Fisher's exact tests for categorical variables and Student's $t$-test for continuous variables. Level of significance was considered at a $P$-value of less than 0.05 at the $95 \%$ level of confidence interval.

\section{Ethical considerations}

The Research and Publications Committee of the Muhimbili University of Health and Allied Sciences granted ethical clearance for the study. Permission was sought from the respective hospital administrations to access the PMTCT register books for the purpose of carrying out this study. Before being asked to participate in the study, participants were given a description of the study and provided written informed consent.

\section{Results}

\section{Review of patients files}

A total of 498 children whose data had been recorded for the past 2 years in the data registry were enrolled into the study. Review of the records indicated that all infants who were registered in the PMTCT register books were born to confirmed HIV-infected mothers. Age at which co-trimoxazole prophylaxis was initiated was recorded as documented. Dosages and duration of co-trimoxazole use were also recorded from the registry.

The results show that there was a very large variation of the age at which co-trimoxazole prophylaxis was initiated. Majority of infants were initiated prophylactic therapy at the age of 3 months. Out of 498 children who were initiated on co-trimoxazole prophylaxis, more than $80.3 \%$ of them were initiated within 2 months of birth. Only 291 (58.4\%) children were provided with co-trimoxazole prophylaxis within 4 weeks after birth. Three hundred ninety-seven $(79.7 \%)$ children received co-trimoxazole prophylaxis within 3 months after birth, whereas 31 children $(6.2 \%)$ received co-trimoxazole prophylaxis $\geq 6$ months after birth (Table 2).

Doses were given as either $2.5 \mathrm{~mL}(120 \mathrm{mg})$ or $5 \mathrm{~mL}$ (240 mg) of co-trimoxazole syrups. Therefore, calculation of doses was made for conversion into the strength (mg) of co-trimoxazole. Majority $(n=458,91.9 \%)$ of children received $120 \mathrm{mg}$ of co-trimoxazole per day, whereas 39 $(7.8 \%)$ received $240 \mathrm{mg} / \mathrm{day}$, and one $(0.2 \%)$ child received 
Table 2 Age at which co-trimoxazole prophylaxis was initiated in children as recorded from infant files $(n=498)$

\begin{tabular}{lll}
\hline $\begin{array}{l}\text { Age when co-trimoxazole } \\
\text { was initiated (months) }\end{array}$ & $\begin{array}{l}\text { Number of } \\
\text { children }\end{array}$ & Percentage \\
\hline 1 & 291 & 58.43 \\
2 & 105 & 21.1 \\
3 & 1 & 0.2 \\
4 & 50 & 10.04 \\
5 & 10 & 2.0 \\
6 & 6 & 1.2 \\
7 & 11 & 2.21 \\
8 & 7 & 1.41 \\
9 & 2 & 0.4 \\
10 & 5 & 1.0 \\
11 & 2 & 0.41 \\
12 & 4 & 0.8 \\
$\geq 12$ & 4 & 0.8 \\
Total & 498 & 100 \\
\hline
\end{tabular}

$480 \mathrm{mg} /$ day. There was no recorded patient's weight in the PMTCT register books. It was therefore not possible to correlate the prescribed dose with children's body weights.

In this study, 126 (25.3\%) children received co-trimoxazole prophylaxis based on unconfirmed HIV-infection for the duration of 6-8 months. One hundred and eleven (22.3\%) children received co-trimoxazole prophylaxis for less than 3 months before confirmation of their serostatus (Table 3).

There was no documentation of children tolerance to cotrimoxazole, adherence, side effects, and drug interactions. We could therefore not establish how well the infants tolerated the prescribed doses of co-trimoxazole based on the available records.

\section{Interviews of parents and guardians for children at the health facilities}

A total of 321 parents and guardians were interviewed; 169 (52.6\%) being females and 152 (47.4\%) males. Parents and guardians were interviewed at Muhimbili National Hospital (38.6\%), Temeke (25.5\%), Amana (15.9\%), and Mwananyamala (19.9\%) hospitals. Table 4 shows the age of

Table 3 Duration of co-trimoxazole use for prophylaxis of opportunistic infections among children as documented from patient files in the health facilities $(n=498)$

\begin{tabular}{lll}
\hline Duration (months) & Number of children & Percentage \\
\hline$<3$ & 111 & 22.3 \\
$3-5$ & 97 & 19.5 \\
$6-8$ & 126 & 25.3 \\
$9-12$ & 100 & 20.0 \\
$>12$ & 64 & 12.9 \\
Total & 498 & 100 \\
\hline
\end{tabular}

Table 4 Age distribution of the children whose parents/guardians were interviewed at the health facilities regarding provision of cotrimoxazole prophylaxis $(n=32 I)$

\begin{tabular}{lll}
\hline Age (months) & $\begin{array}{l}\text { Number of parents/ } \\
\text { guardians }\end{array}$ & Percentage \\
\hline $1-4$ & 84 & 26.2 \\
$5-9$ & 164 & 51.1 \\
$10-14$ & 61 & 19.0 \\
$14-19$ & 11 & 3.4 \\
$\geq 20$ & 1 & 0.3 \\
Total & 321 & 100 \\
\hline
\end{tabular}

the children who were receiving co-trimoxazole prophylaxis in the CTCs as reported by parents and guardians.

Parents and guardians were interviewed about adherence to co-trimoxazole prophylaxis for their children. Forty-two parents and guardians (13.1\%) reported that their children have had missed one or more doses of cotrimoxazole during the period of prophylaxis. Fifteen (4.7\%) parents and guardians reported that their children had missed doses of co-trimoxazole prophylaxis during the past 1 month at the time of interview, whereas seven $(2.2 \%)$ children had missed co-trimoxazole for the past 2 weeks. Alteration of dosage regimen by decreasing the frequency of use of the prescribed dosages was reported by a small proportion (7.9\%) of parents (Table 5). The main reasons for altering the doses of co-trimoxazole by parents and guardians were due to the perceived co-trimoxazole side effects or when the children were feeling unwell.

Based on the results and criteria used to assess adherence as shown in Table 5, an assessment was made to determine the adherence level of co-trimoxazole prophylaxis among children in the four health facilities. Proportions of children who skipped doses of co-trimoxazole were $26.2 \%$ at Muhimbili, $33.3 \%$ at Amana, $19.0 \%$ at Mwananyamala, and $21.4 \%$ at Temeke hospitals. Therefore, results show no significant differences between the four hospitals in terms of adherence to co-trimoxazole prophylaxis in children $(P>0.05)$.

Table 5 Proportion of children who missed co-trimoxazole prophylaxis as reported by parents and guardians $(n=42)$

\begin{tabular}{lll}
\hline $\begin{array}{l}\text { Duration of non- } \\
\text { adherence to co- } \\
\text { trimoxazole prophylaxis }\end{array}$ & $\begin{array}{l}\text { Number of parents/ } \\
\text { guardians }\end{array}$ & Percentage \\
\hline Past 3 days & 15 & 4.7 \\
Past 7 days & 5 & 1.6 \\
Past I4 days & 7 & 2.2 \\
Past 30 days & 15 & 4.7 \\
Total non-adherent & 42 & 13.1 \\
\hline
\end{tabular}


All parents and guardians reported that co-trimoxazole for prophylaxis of opportunistic infections in children is provided for free in the hospitals. More than one-third (36.1\%) of parents and guardians reported lack of refrigerators as the main challenge for the provision of co-trimoxazole prophylaxis to children at home. Lack of pediatric co-trimoxazole formulation in the hospitals $(9.7 \%)$ and difficulties in understanding how to use the drug $(9.3 \%)$ were other challenges reported by parents and guardians. Lack of transport facilities to attend scheduled clinic visits was the case reported by $16(5.0 \%)$ parents and guardians. More than half $(67 \%)$ of parents or guardians reported that they were provided with pediatric co-trimoxazole formulations during all the scheduled visits at the health facilities. On the other hand, $33 \%$ of parents and guardians reported to have missed co-trimoxazole at the health facilities in one or more occasions. Whenever the drug was not available at the hospital, $54.8 \%$ of parents and guardians bought the drug from private pharmacies, while 19.9\% could not afford the price in the pharmacies and therefore had to wait until when the next available in the hospitals.

Some parents and guardians reported to have observed co-trimoxazole-induced side effects among their children. Out of all children, 28 (8.7\%) of them were reported to have experienced some side effects related to co-trimoxazole use. The most frequently experienced side effects were nausea and vomiting (3.4\%), and hypersensitivity reactions (3.4\%). Majority $(86.7 \%)$ of parents and guardians had a positive perception toward co-trimoxazole prophylaxis indicating that it is effective in preventing opportunistic infections in children. Of the parents and guardians interviewed, 42 (13.1\%) were not sure of the effectiveness of the drug, whereas only a very small proportion $(0.6 \%)$ indicated that co-trimoxazole prophylaxis is not effective in preventing opportunistic infections in children.

\section{Interview of health care workers}

There were a total of 126 health care workers interviewed in the study. There were $50(39.7 \%)$ health care workers from Muhimbili, 26 (20.6\%) from Mwananyamala, 25 (19.8\%) from Amana, and 25 (19.8\%) from Temeke hospitals. Out of 126 health care workers, $49(38.9 \%)$ were males and $77(61.1 \%)$ were females. Table 6 presents the designation of the health care workers who were interviewed in the study.

More than half $(66.7 \%)$ of the health care workers reported to have experienced unavailability of co-trimoxazole pediatric formulations in the health facilities. Approximately one-third (28.6\%) of the health care workers reported scarcity of the drug from the central medical stores department
Table 6 Designation of the health care workers who were interviewed regarding provision of co-trimoxazole prophylaxis in children born to HIV-infected mothers in the health facilities $(n=126)$

\begin{tabular}{lll}
\hline Designation & $\begin{array}{l}\text { Number of health } \\
\text { care workers }\end{array}$ & Percentage \\
\hline Medical doctors & 25 & 19.8 \\
Assistant medical officers & 10 & 7.9 \\
Clinical officers & 8 & 6.3 \\
Pharmacists & 13 & 10.3 \\
Pharmacy technicians & 3 & 2.4 \\
Nursing officers & 54 & 42.9 \\
Counselors & 7 & 5.6 \\
Others & 6 & 4.8 \\
Total & 126 & 100 \\
\hline
\end{tabular}

as the main reason for such a shortage. Others (19.0\%) reported a lack of funds to purchase essential medicines in the hospitals as the main attributing factor for experiencing such shortages.

In the absence of co-trimoxazole syrup, approximately one-quarter $(23.8 \%)$ of health care workers gave instructions to parents and guardians to prepare co-trimoxazole suspension using crushed tablets at home. Some health care workers $(17.5 \%)$ were able to assist parents and guardians by making co-trimoxazole suspension using co-trimoxazole tablets. Others $(19.8 \%)$ requested parents and guardians to come back to the health facilities the next day with anticipation that the drug will be available during that time. Majority (59.5\%) of health care workers indicated that lack of co-trimoxazole syrups plays a major role in poor adherence to the medication among children.

An assessment was also made about the factors which are considered by health care workers as a prerequisite for the initiation of co-trimoxazole prophylaxis to children born to HIV-infected mothers at the health facilities. Weight and age of the child, commitment of parents and guardians to administer co-trimoxazole to a child at home, confirmed HIV status of the mother and commitment of parents or guardians to follow the scheduled visits to the clinics were among the factors considered by the health care workers before initiation of co-trimoxazole prophylaxis (Table 7).

Health care workers mentioned a number of challenges as barriers for effective implementation of co-trimoxazole prophylaxis in children. These included lack of pediatric co-trimoxazole formulations (20.6\%), unavailability of upto-date treatment guidelines for co-trimoxazole prophylaxis (19.0\%), difficulties in the determination of the weights of children for calculation of doses (5.6\%), and heavy patient load (56.3\%). 
Table 7 Factors considered by health care workers before initiation of co-trimoxazole prophylaxis to children born to HIVinfected mothers at the health facilities $(n=126)$

\begin{tabular}{|c|c|c|}
\hline Factor & $\begin{array}{l}\text { Number of } \\
\text { health care } \\
\text { workers }\end{array}$ & Percentage \\
\hline Weight of the child & 32 & 25.4 \\
\hline Age of the child & 87 & 69.0 \\
\hline $\begin{array}{l}\text { Commitment of parents/guardians to } \\
\text { administer the drug to a child at home } \\
\text { as instructed }\end{array}$ & 10 & 7.9 \\
\hline Confirmed HIV status of the mother & 50 & 39.7 \\
\hline $\begin{array}{l}\text { Commitment of parents/guardians to } \\
\text { follow the scheduled visits to the clinic }\end{array}$ & 7 & 5.6 \\
\hline $\begin{array}{l}\text { Availability of pediatric co-trimoxazole } \\
\text { formulations at the health facility }\end{array}$ & 10 & 7.9 \\
\hline Counseling of parents and guardians & 47 & 37.3 \\
\hline
\end{tabular}

Majority of health care workers (89.7\%) pointed out that co-trimoxazole is very effective against many opportunistic infections in children. Health care workers (39.5\%) also reported that lack of knowledge among parents and guardians regarding the use of co-trimoxazole is a contributing factor to failure in adherence to the medication. Forty-two (33.3\%) health care workers reported the shortage of co-trimoxazole in the health facilities as the main challenge affecting provision of the drug to children. On the other hand, 33 (26.2\%) health care workers reported negative attitude of parents and guardians regarding the use of co-trimoxazole for the prevention of opportunistic infections in children as one of the impediments for effective implementation of co-trimoxazole prophylaxis in children born to HIV-infected mothers.

\section{Discussion}

In this study, challenges encountered in the provision of cotrimoxazole prophylaxis in children born to HIV-infected mothers were assessed through the review of PMTCT files and interviews of caregivers of children and health care providers in the public hospitals. Tanzanian National AIDS Control Program guidelines stipulate that all infants born to HIV-infected mothers should be given co-trimoxazole prophylaxis to prevent opportunistic infections until when their HIV status is confirmed at 18 months of age. ${ }^{13}$ In this study, records of 498 children born to HIV-infected mothers were assessed for the time of initiation, dosage, and duration of the use of co-trimoxazole prophylaxis before confirmation of the children's HIV status.

According to the guidelines, children born to HIV-infected mothers should be initiated co-trimoxazole prophylaxis from 4 to 6 weeks after birth. ${ }^{13}$ In this study, approximately $40 \%$ of infants were initiated prophylaxis much later (8-48 weeks) after birth. To effectively manage opportunistic infections in children, it is necessary to identify the reasons for delayed initiation of co-trimoxazole prophylaxis in eligible children. Some of these factors are facility-based such as unavailability of the drug, weighing scales to determine the weights of children for dose calculation, and heavy patient load. ${ }^{18,16}$ As reported by health care workers, other reasons on why some children are not started on co-trimoxazole prophylaxis on time include lack of commitments of parents and guardians to ensure strict adherence of children to the prescribed dosage of co-trimoxazole. This is one of the main reasons on why some health care workers are hesitant to initiate co-trimoxazole prophylaxis in HIV-exposed children.

Availability of pediatric co-trimoxazole oral formulations is a crucial factor for effective implementation of cotrimoxazole prophylaxis in children. Although in Tanzania the drug is provided for free, most of the public health facilities have frequent stock-outs of essential medicines including co-trimoxazole. ${ }^{19,20}$ The main factors attributable to this scarcity are unavailability of medicines from the government suppliers in this case the medical stores department and lack of funds to purchase medicines at the health care facility level. ${ }^{21}$ Based on these results, it is necessary that supply management of essential medicines including co-trimoxazole should be well coordinated to ensure availability. Training of health care providers and sensitization of communities to ensure that children born to HIV-infected mothers are referred to the health facilities is also necessary for effective implementation of this policy.

Although co-trimoxazole prophylaxis is very effective for prevention of opportunistic infections in HIV-exposed children, ${ }^{22}$ the findings of this study cannot guarantee its effectiveness. Due to frequent stock out of co-trimoxazole in the health facilities, a substantial number of parents and guardians of children reported that their children had missed one or more doses during the time of prophylaxis. Moreover, unavailability of co-trimoxazole syrups in the health facilities made it difficult for parents and guardians to administer accurate doses to children using crushed tablets. In addition, the doses were prescribed based on the age of children rather than the weight. It is therefore not known whether the children who are HIV-infected did get appropriate doses to prevent opportunistic infections.

For co-trimoxazole to be effective in prevention of opportunistic infections in children, adherence to the medication is essential. ${ }^{23}$ In this study, there was no documentation in the infants' files about the protective effects of co-trimoxazole 
against opportunistic infections in children. A recent study conducted in the same hospitals in Dar es Salaam indicated a high prevalence rate of Escherichia coli resistance to cotrimoxazole in HIV-infected adult patients. ${ }^{24}$ In that study, it was reported that non-adherence to co-trimoxazole prophylaxis among patients may have had contributed to the observed E. coli resistance to co-trimoxazole. ${ }^{24}$ In this study, due to the fact that a high proportion of children missed the prescribed doses of co-trimoxazole for varied period of time during prophylaxis, emergence of resistance to bacteria and other pathogens to co-trimoxazole cannot be ruled out in this group of individuals.

Studies have reported that co-trimoxazole is safe and cost-effective for the prevention of opportunistic infections in HIV-infected individuals. ${ }^{25}$ Since there was no documentation of co-trimoxazole-induced side effects in the infants' files, it is not known to what extent the children tolerated the doses of co-trimoxazole. However, reports from parents and guardians who were interviewed indicated that there were no major side effects experienced by children during the course of prophylaxis. The reported side effects were mild and self-limiting in majority of the children. These included gastrointestinal side effects such as nausea and vomiting and dermatological conditions; itching and hypersensitivity reactions. These findings are in line with those reported elsewhere about the safety profile of co-trimoxazole when used for prophylaxis of opportunistic infections. ${ }^{26}$

Confirmed HIV status of the mother and commitment of parents or guardians to administer co-trimoxazole at home and subsequent follow-up for visits to the clinics by parents and guardians have been implicated in the long-term effectiveness of co-trimoxazole prophylaxis for opportunistic infections. ${ }^{27,28}$ Although most health care workers pointed out that knowing the weight of the child was important to guide dosage determination, this was rarely practiced in the hospitals. There was also no documentation of the age of patients at each visit and hence there was no clear distinction of the dosages between age groups as required by the guidelines. ${ }^{13}$ The main reasons for not recording children's weight were heavy patient work load, lack of sufficient time for counseling patients, and unavailability of weighing scales in the hospitals. Thus, weight-specific dosages and related guidelines need to be adopted along with the availability of the required facilities and pediatric oral formulations of cotrimoxazole in the hospitals.

Co-trimoxazole prophylaxis should be continued for a sufficient period of time until when the child is no longer exposed to maternal antibodies or proved negative, which could be done at 4 weeks using PCR or at 18 months of age using an antibody test. In this study, approximately one-quarter of all children were provided presumptive co-trimoxazole prophylaxis for 6-8 months before being diagnosed. Only a small proportion of children $(22.3 \%)$ received the drug for less than 3 months before being diagnosed. These findings reflect the diagnostic and related challenges in the public hospitals mainly due to unavailability of diagnostic facilities and other logistical support for diagnosis of HIV-exposed children.

In this study, some children $(1.6 \%)$ were continued on co-trimoxazole prophylaxis 1 year after birth. Due to unavailability of diagnostic facilities and failure to ascertain the serostatus of children born to HIV-infected mothers, other studies have also recommended presumptive diagnosis to be done for at least 1 year in resource-poor settings. ${ }^{12}$ These findings emphasize the fact that to provide sufficient prevention against opportunistic infections, co-trimoxazole prophylaxis need be provided for sufficient time before a correct diagnosis can be made to determine the serostatus of the child.

Perception of health care workers, parents, and guardians is an important aspect in terms of management policy for HIV-exposed children. Co-trimoxazole was reported to be very effective in preventing opportunistic infections by most health care workers, parents, and guardians. This observation supports scaling up of co-trimoxazole prophylaxis program to lower level health facilities to reach the needy and combat HIV and its accompanying complications. However, lack of knowledge among parents or guardians regarding the use of co-trimoxazole prophylaxis and shortage of pediatric co-trimoxazole formulations and diagnostic facilities in the health facilities need to be addressed before scaling up of this program can be successfully implemented.

\section{Conclusion}

The results from this study have identified the key challenges for effective implementation of co-trimoxazole prophylaxis in HIV-exposed children in the public health facilities. These findings are important for health planners and policy makers such as the Ministry of Health and Social Welfare through the National AIDS Control Program as they plan to scale up co-trimoxazole prophylaxis to lower level health facilities. In this study, most children who were using co-trimoxazole prophylaxis had no major opportunistic infections and the drug was well tolerated. The major barrier to compliance was unavailability of pediatric co-trimoxazole formulations in the health facilities. Since co-trimoxazole prophylaxis is relatively inexpensive, safe, effective, and reduces morbidity 
especially in children infected with HIV, the identified challenges should be urgently addressed to optimize its use.

\section{Acknowledgments}

The authors thank the Muhimbili University of Health and Allied Sciences for providing financial support for this study. We extend special appreciation to the management of Muhimbili National Hospital and regional hospitals for providing access to conduct the study in the hospitals. We are grateful to the medical practitioners, nurses, and drug dispensers for participating in the study. Special thanks to the parents and guardians of children for agreeing to participate in the interviews.

\section{Disclosure}

The authors report no conflicts of interest in this work.

\section{References}

1. Tanzania Commission for AIDS. UNAIDS Report on Country Progress Reporting (Tanzania Mainland). Dar es Salaam: Tanzania Commission for AIDS; 2012.

2. Fischl MA, Dickinson GM, La Voie L. Safety and efficacy of sulfamethoxazole and trimethoprim chemoprophylaxis for Pneumocystis carinii pneumonia in AIDS. JAMA. 1988;259:1185-1189.

3. Graham SM. Prophylaxis against Pneumocystis carinii pneumonia for HIV-exposed infants in Africa. Lancet. 2002;360:1966-1968.

4. Graham SM, Mtitimila EI, Kamanga HS, Walsh AL, Hart CA, Molyneux ME. Clinical presentation and outcome of Pneumocystis carinii pneumonia in Malawian children. Lancet. 2000;355:369-373.

5. Gonzalez D, Melloni C, Poindexter BB, et al. Stimulations determination of trimethoprim and sulfamethoxazole in dried plasma and urine spots. Bioanalysis. 2015;7(9):1137-1149.

6. Brumfitt W, Hamilton-Miller JM. Limitations of and indications for the use of co-trimoxazole. J Chemother. 1994;6:3-11.

7. Wiktor SZ, Sassan-Morokro M, Grant AD, et al. Efficacy of trimethoprim-sulphamethoxazole prophylaxis to decrease morbidity and mortality in HIV-1-infected patients with tuberculosis in Abidjan, Cote d'Ivoire: a randomized controlled trial. Lancet. 1999;353: 1469-1475.

8. Mermin J, Lule J, Ekwaru JP, et al. Effect of co-trimoxazole prophylaxis on morbidity, mortality, CD4-cell count and viral load in HIV infection in rural Uganda. Lancet. 2004;364:1428-1434.

9. Thera MA, Sehdev PS, Coulibaly D, et al. Impact of trimethoprimsulfamethoxazole prophylaxis on falciparum malaria infection and disease. J Infect Dis. 2005;192:1823-1829.

10. WHO/UNAIDS. Joint WHO/UNAIDS/UNICEF Statement on Use of Cotrimoxazole as Prophylaxis in HIV Exposed and HIV Infected Children. Geneva: WHO/UNAIDS; 2004. Available from: http://www. who.int/3by5/mediacentre/en/Cotrimstatement.pdf. Accessed March 8, 2015.

11. Gill CJ, Mwanakasale V, Fox MP, et al. Effect of presumptive co-trimoxazole prophylaxis on pneumococcal colonization rates, seroepidemiology and antibiotic resistance in Zambian infants: a longitudinal cohort study. Bull World Health Organ . 2008;86(12):929-938. doi:102471/BLT 049668.
12. Grimwade K, Swingler GH. Cotrimoxazole prophylaxis for opportunistic infections in children with HIV infection. Cochrane Database of Syst Rev. 2003;1:CD003508. doi:10.1002/14651858.CD003508.pub2.

13. Tanzanian National AIDS Control Program. National Guidelines for the Clinical Management of HIV and AIDS. 4th ed. Dar es Salaam: Tanzanian National AIDS Control Program; 2012.

14. WHO/UNICEF. Co-trimoxazole prophylaxis for HIV-exposed and HIVinfected infants and children: Practical approaches to implementation and scale up. World Health Organization and INICEF; 2009. Available from: http://www.unicef.org/aids/files/CotrimoxazoleGuide_2009.pdf. Accessed August 2015.

15. Feucht UD, Meyer A, Kruger M. Missing HIV prevention opportunities in South African children - a 7-year review. BMC Public Health. 2014; $14: 1265$.

16. Silumbe S, Kamuhabwa AR. Management of antimalarial drugs in the urban public health facilities in Tanzania. Int J Pharm Sci Res. 2015;6: $154-163$.

17. Brou H, Desgrées-du-Loû A, Souville M, Moatti J-P, Msellati P; Initiative Evaluation Group in Côte d'Ivoire. Prophylactic use of cotrimoxazole against opportunistic infections in HIV-positive patients: knowledge and practices of health care providers in Côte d'Ivoire. AIDS Care. 2003;15:629-637.

18. Kamuhabwa AR, Silumbe R. Knowledge among drug dispensers and antimalarial drug prescribing practices in public health facilities in Dar es Salaam. Drug Healthc Patient Saf. 2013;5:181-189.

19. Kamuhabwa A, Saifuddin M, Maganda B, Kaale E. Quality of cotrimoxazole tablets used for management of opportunistic infections in HIV/AIDS patients. Int J Pharm Res. 2011;3:70-75.

20. Yohana E, Kamuhabwa A, Mujinja P. Availability and affordability of anticancer medicines at the Ocean Road Cancer Institute in Dar es Salaam, Tanzania. East Afr J Public Health. 2011;8:52-57.

21. Date AA, Vitoria M, Granich R, Banda M, Fox MY, Gilks C. Implementation of co-trimoxazole prophylaxis and isoniazid preventive therapy for people living with HIV. Bull World Health Organ. 2010;88: 253-259.

22. Homsy J, Dorsey G, Arinaitwe E, et al. Protective efficacy of prolonged co-trimoxazole prophylaxis in HIV-exposed children up to age 4 years for the prevention of malaria in Uganda: randomized controlled openlabel trial. Lancet Glob Health. 2014;2:e727-e736.

23. Walker S, Ford D, Mulenga V, et al. Adherence to both cotrimoxazole and placebo is associated with improved survival among HIV-infected Zambian children. AIDS Behav. 2009;13(1):33-41.

24. Mwambete KD, Kamuhabwa AR. Resistance of commensal intestinal Escherichia coli and other enterics to co-trimoxazole and commonly used antibiotics in HIV/AIDS patients. Clin Microbiol. 2013;3:134.

25. Ryan M, Griffin S, Chitah B, et al. The cost-effectiveness of cotrimoxazole prophylaxis in HIV-infected children in Zambia. AIDS. 2008; 22(6):749-757.

26. Watera C, Todd J, Muwonge R, et al. Feasibility and effectiveness of cotrimoxazole prophylaxis for HIV-1-infected adults attending an HIV/AIDS clinic in Uganda. J Acquir Immune Defic Syndr. 2006;42:373-378.

27. Madhi SA, Petersen K, Madhi A, Khoosal M, Klugman KP. Increased disease burden and antibiotic resistance of bacteria causing severe community-acquired lower respiratory tract infections in human immunodeficiency virus type 1-infected children. Clin Infect Dis. 2000;31: 170-176.

28. Lin D, Li W-K, Rieder MJ. Cotrimoxazole for prophylaxis or treatment of opportunistic infections of HIV/AIDS in patients with previous history of hypersensitivity to cotrimoxazole. Cochrane Database Syst Rev. 2007;2:CD005646. 
Drug, Healthcare and Patient Safety

Dovepress

\section{Publish your work in this journal}

Drug, Healthcare and Patient Safety is an international, peer-reviewed open-access journal exploring patient safety issues in the healthcare continuum from diagnostic and screening interventions through to treatment, drug therapy and surgery. The journal is characterized by the rapid reporting of reviews, original research, clinical, epidemiological and

post-marketing surveillance studies, risk management, health literacy and educational programs across all areas of healthcare delivery. The manuscript management system is completely online and includes a very quick and fair peer-review system. Visit http://www.dovepress.com/ testimonials.php to read real quotes from published authors.

Submit your manuscript here: http://www.dovepress.com/drug-healthcare-and-patient-safety-journal 